\title{
Instrumental evaluation of traction machines sparking
}

\author{
Nikita Kolobashkin ${ }^{1}$, Beligma Dorzieva ${ }^{1}$, Evgeniy Beyerleyn ${ }^{1, *}$, Polina Tyuteva, and \\ Anatoliy Cukublin ${ }^{1}$
}

${ }^{1}$ National Research Tomsk Polytechnic University, 634050 Tomsk, Russia

\begin{abstract}
In the article, the authors considered the possibility of the sparking monitoring at a commutator of a traction motor under operating conditions. The authors developed a method for the evaluation of sparking level at the commutator by means of the current flowing through separate, isolated parts of the split brush recording. The relationship between an auxiliary commutation current and the sparkling level is revealed.
\end{abstract}

\section{Introduction}

The importance of brushed direct current traction motors (DC TM) on electrified sections of the railway has not decreased in recent years, as it was expected, but on the contrary began to grow. There have appeared new electric locomotives 2ES4, 2ES5, 2ES6 and others, intended for the replacement of outmoded types of machines constructed on the basis of DC machines [1]. At the same time, there are problems related to the commutator unit, monitoring of its quality and magnetic system maintenance at the depot test stations. Tests carried out on the locomotives equipped with DC motor shows that reliability is also affected by design flaws as well as drawbacks associated with control circuits [2].

The failure analysis shows that about $90 \%$ of DC TM damages occur at about 800 thous. km run from the factory repairs, and approximately $30 \%$ of the DC TM fail during the warranty period at the established mileage per one cycle from the beginning of operation (or overall repair) to the next overall repair 1400 thous.km. As experience has shown one of the effective ways to reduce the number of failures is to monitor the DC TM condition after repair and during operation [3]. However, if testing is performed at repair depot there are National standards and rules for machines testing, then for the testing directly on the electric locomotive there are neither normative documents for controlling the DC TM condition during operation, nor technical facilities for its implementation (except armature current measurement). The availability of methods and technical facilities for controlling could prevent the DC TM from being installed on the electric locomotive without an appropriate setting of commutation and magnetic system, which initially guarantees a mileage rate under normal operating conditions. Monitoring of the DC TM condition during operation makes possible to regulate the load and apply the technique of thrust redistribution, and effectively prevent the slippage. The presented work is done by

\footnotetext{
* Corresponding author: beierleinev@tpu.ru
} 
the specialists of the Electrotechnical Complexes and Materials Department, Tomsk Polytechnic University and devoted to the creation of method and technical facilities for the DC TM condition monitoring at a repair depot and on an electric locomotive.

\section{Theoretical study}

The main indicator of the DC TM is the commutator sparking. According to the commutation theory, there will be no sparking if the commutation is linear and the additional commutation current is zero. If the machine has overcommutation or undercommutation, this current differs from zero value. It flows in the section closed with a brush, and flows through the brush in the cross direction [4]. Therefore, K.I. Shenfer called it as the cross current of the brush. As a result of the studies, a quantitative relationship was established between the magnitude of the additional commutation current and the sparking energy. The form of the additional commutation current was investigated as on the developed mathematical model, as well as on the real traction motor, that contributed on a measured signal processing technique. The estimation of the armature and excitation currents influence, the armature speed, brush contact area, commutator temperature and other performance parameters of DC traction motor made it possible to create a new method for the sparking evaluation which is based on additional commutation current measuring and correlation with sparking level in accordance with National Standard GOST 183- 74 .

The main point of the method involves recording the cross current and identifying the relation with the sparking level of the commutator unit. The application of this method is most convenient when the motor brush is split (for example, TL2K1, NB-514, DPT810U), as for cross current measuring no change in the commutator design is required. Consider the fact that electromagnetic processes in the machine influence the performance of the commutation process, thermal and mechanical, the value and waveform of the additional commutation current should be construed as a universal characteristic of the electric motor condition [5].

To measure the commutation current, a spark monitoring device (SCR) was used, this device consists of a sensor made on the basis of stock-produced split brushes 1 and 2 that are isolated from each other (Fig. 1), a converter device 3 and a signal measuring system consisting of an analog-to-digital converter. The output of signal measuring system is connected to a personal computer. Unlike the well-known devices, for example PKK2, PKK5, this spark monitoring device uses standard composite motor brush in a function of sensitive element, the sparking on this brush-commutator unit is required to be determined. As a primary transformer we use a specially designed current transformer. The brush is connected to this current transformer so that an additional commutation current flows through its primary winding, the frequency of this current depends on the armature speed and the number of commutator segments. The current transformer is mounted on the brush holder. Its installation on brush holder does not cause any difficulty on any direct current traction or auxiliary motor. The secondary winding of the current transformer is connected to an analog-to-digital converter, which in turn is connected to a computing system. As computing system a portable PC with a specialized computing system or a special controller with appropriate memory can be used. The connection diagram is shown on Fig. 1.

A main feature of the spark monitoring device design is (Fig. 2):

1) the galvanic isolation of the measuring circuit and DC TM supply circuits, which makes the operation with the spark monitoring device safe;

2) the absence of an additional power source of the sensor installed in the traction motor, which dramatically simplifies the measurement circuit. 


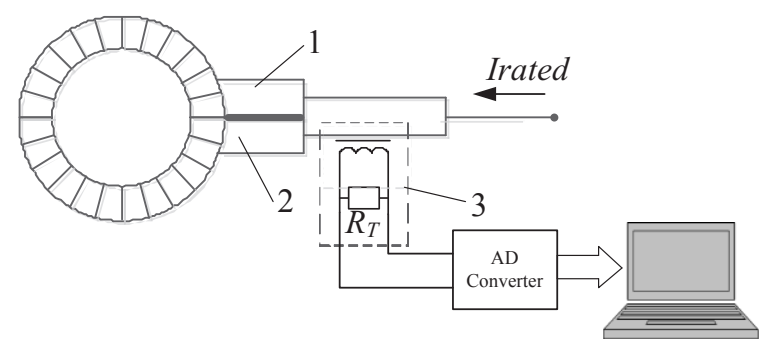

Fig. 1. Connection diagram of spark monitoring device and motor.

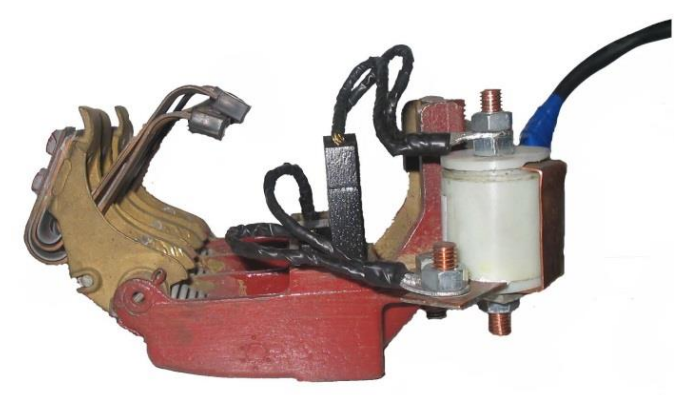

Fig. 2. The exterior of the spark monitoring device and its installation on the brush holder.

\section{Experimental study}

To determine the sparking intensity, the value of the additional commutation current was taken across the load resistor $\left(R_{T}\right)$ of the measuring current transformer mounted on the brush holder. According to the energy theory of commutation [3], the excess of the measured current variable component over a certain acceptable value leads to the sparking appearance. It is known that the sparking intensity or sparking level is determined by the power segregated under the brush per length $L_{b r}$ in the time $t_{k} / \vartheta_{k},\left(\right.$ tk is the segment pitch, $\vartheta_{k}$ is the commutator peripheral velocity) $[4,6]$ :

$$
\Delta P=\frac{L_{c} i_{k}^{2}}{2} * \frac{\vartheta_{k}}{t_{k} L_{b r}}
$$

According to this equation, if the commutation current $i_{k}$ is recorded for a particular electrical machine, the power $\Delta P$ segregated under the brush will be known; its magnitude can determine the occurrence and sparking level on the commutator. During the tests carried out on experimental model of the traction motor, the cross current curves (Fig. 3) flowing between the individual parts of the split brush were recorded. The sparking intensity was determined visually according to National Standard GOST 183-74.

Next comes the parallel processing of signals, in which two problems need to be solved: the determination of the sparking level and the degree of commutation adjustment. For spark indication, the effective value of the signal is calculated using the RMS method, then the signal is calibrated by recalculating the data on the calibration curve. The result is displayed on the front panel of the devices on the computer screen as shown in Fig. 4. These data are information on the sparking level on the commutator and are graded in accordance with National Standard GOST 183-74. To adjust the commutation, it is necessary to analyze the array of instantaneous values of the spark monitoring device. 


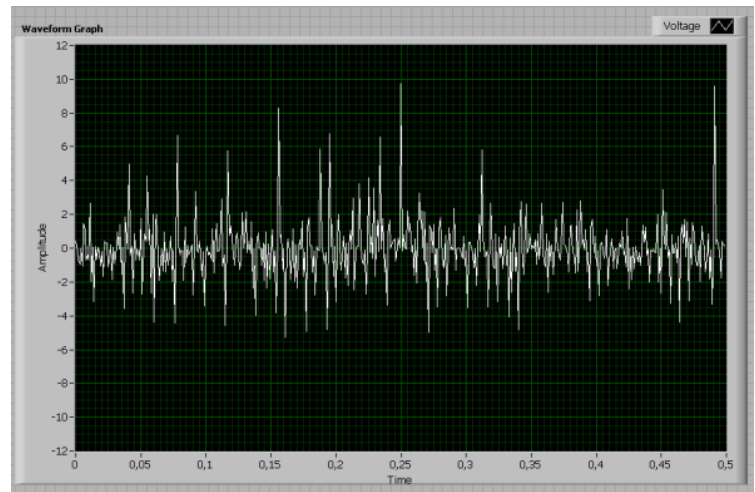

Fig. 3. Cross current curve.

From this array, at each two points, the minimum and maximum values of the signal are calculated, after calculating its absolute value is taken and compared between the minimum and maximum. If the maximum value is greater than the minimum value, the signaling information "Overcommutation" is presented on the front panel, if the condition is not met, Virtual instruments panel signals "Undercommutation" (Fig.4). The difference between the absolute values of the maximum and minimum is displayed on the virtual panel in the form of a pointer device with indication in per unit values. When carrying out a series of tests for each type of traction motor, it is possible to calibrate the indication of this device in millimeters of the diamagnetic gap under the interpoles that significantly simplify the magnetic system tuning. In the suggested study, only a qualitative assessment of the general commutation type is possible. Next, the algorithm implements the test report recording. For this, it is necessary to ensure that only the values specified in National Standard GOST 18374 are recorded in the test report.

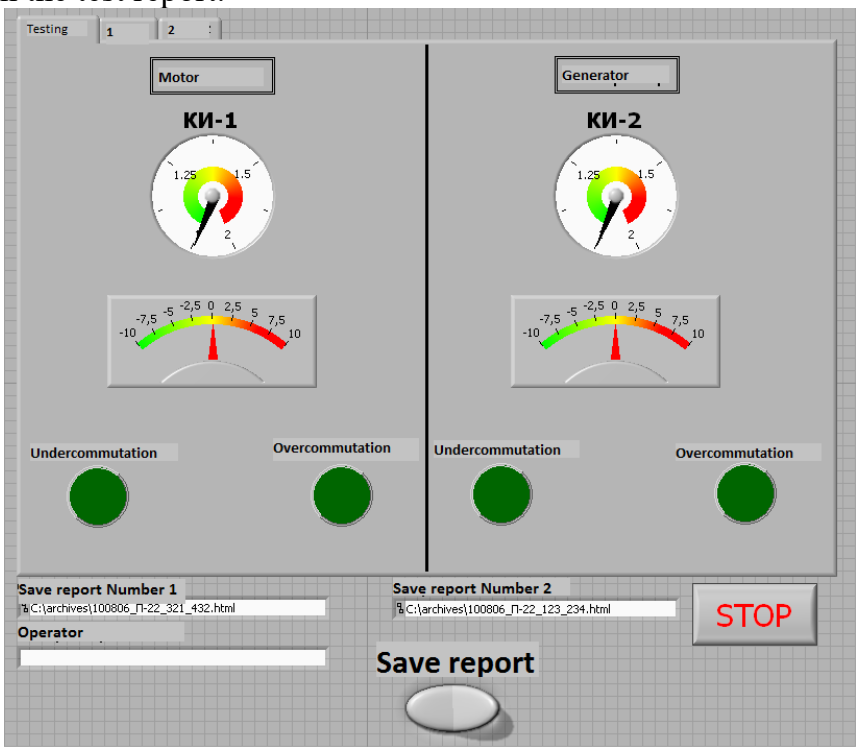

Fig. 4. Virtual instruments panel.

The pilot installation of the spark monitoring device at the test stations of the Ulan-Ude Locomotive and rail-way car repair plant, the Taiga locomotive repair depot of the West Siberian Railroad, and the Ural Locomotives shows that the monitor of sparking level of the DC TM is carried out not only in commutation tests but also in other test modes . It is 
carried out automatically with normally closed assembling hatches. Sensors installation takes about $10 \ldots 15$ minutes. The recording of the sparking level in grades in the protocol is performed by the operator command.

\section{Discussion}

The suggested spark monitoring device on direct current traction motors can be used not only at test stations, but also during operation, which makes it possible to use the information obtained for diagnosing and automated DC TM control not only for new locomotives testing at depot but also for monitoring during operation. The additional commutation current change that measured by the spark monitoring device has complex waveform. Studies show that data obtained from spark monitoring device benefit not only the determination of sparking level according to the cross current magnitude, but also provide other important indicators of the DC TM operation, based on the waveform analysis, frequency characteristics, and others [6].

\section{Conclusion}

As follows from the provided study, the commutation tests and monitoring technology of traction and auxiliary motors of electric locomotives are developed and adjusted.

It should also be noted that the wide use of the spark monitoring system and the diagnosis of traction motors is obstructed by the lack of regulations or rules for instrumental sparking detecting. National Standard GOST 2582-81 in the annex of 1991 permits instrumental indication, but this was not reflected in industry Standards. The most important unit of an electric locomotive is a traction electric motor, nowadays it does not have a regulatory framework for the sparking control during operation and diagnosis organization.

\section{References}

1. A.Kotelnikov, A.Nestrahov, Bulletin of VNIIGT, 5, 15 (2000) [in Russian]

2. S.Obuhov, I.Plotnikov, M.Surkov, L.Sumarokova, K.Savkin, MATEC Web Conf. 91, 01036 (2017)

3. V.Shevchuk, O.Muravlev, O.Stolyarova, V.Shevchuk, MATEC Web Conf. 91, 01033 (2017)

4. K.Shenfer, Dymanomachines and DC motors (Enorgoizdat, Moscow, 1927) [in Russian]

5. A. Girnik, A.Fedyanin, A.Kiselev, E.Dorokhina, J. Mash. manuf. and reliab., 46, 3 (2017)

6. D.Shibaev, A.Shibaev, O.Rapoport, A.Cukublin, Sc. Tech. J. Russian Electromecanics, 6, 11-13 (2009) 